\title{
Achieving the Goal of the Fistula First Breakthrough Initiative for Prevalent Maintenance Hemodialysis Patients
}

Janet R. Lynch, PhD,CPHQ,

Mid-Atlantic Renal Coalition, Midlothian, Virginia

Haimanot Wasse, MD,MPH,

Emory University School of Medicine, Atlanta, Georgia

Nancy C. Armistead, MPA, and

Mid-Atlantic Renal Coalition, Midlothian, Virginia

William M. McClellan, MD

Emory University School of Medicine, Atlanta, Georgia

\section{Abstract}

Background-The Centers for Medicare \& Medicaid Services (CMS) established a national goal of 66\% arterio-venous fistula (AVF) use among prevalent hemodialysis (HD) patients for the current Fistula First Breakthrough Initiative (FFBI). The feasibility of achieving the goal has been debated. We examined contemporary patterns of AVF use among prevalent patients to assess the potential for attaining the goal by dialysis facilities and their associated end-stage renal disease (ESRD) Networks in the United States (US).

Study Design—Observational study.

Setting and Participants-US dialysis facilities with a mean HD patient census of 10 or more over the 40 month study period, January 2007 to April 2010.

Outcomes and Measurements-Mean changes in facility-level AVF use and the percent of facilities achieving the $66 \%$ prevalent AVF goal within the US and each Network.

Results-US mean prevalent AVF use within dialysis facilities increased from $45.3 \%$ to $55.5 \%$ $(P<0.001)$ in the US but varied substantially across regions. The percent of facilities achieving the $66 \%$ AVF use goal increased from $6.4 \%$ to $19.0 \%$ ( $P<0.001)$. Over the 40 months, $35.9 \%$ of facilities achieved the CMS goal at least one month. On average, these facilities sustained mean (SD) use of $66 \%$ or greater for 12.9 (11.7) months. Casemix and other facility characteristics explained $20 \%$ of the variation in the proportion of facility patients using an AVF in the last measured month, leaving substantial unexplained variability.

Limitations-This analysis is limited by the absence of facilities' case-mix data over time and the national scope of the initiative precludes use of a comparison group.

(c) 2010 The National Kidney Foundation, Inc. Published by Elsevier Inc. All rights reserved.

Address correspondence to: Janet R. Lynch, PhD CPHQ, Mid-Atlantic Renal Coalition, 1527 Huguenot Road, Midlothian, VA 23113; 804-794-3757; 804-794-3793 (fax); jlynch@nw5.esrd.net..

Publisher's Disclaimer: This is a PDF file of an unedited manuscript that has been accepted for publication. As a service to our customers we are providing this early version of the manuscript. The manuscript will undergo copyediting, typesetting, and review of the resulting proof before it is published in its final citable form. Please note that during the production process errors may be discovered which could affect the content, and all legal disclaimers that apply to the journal pertain.

Financial Disclosure: The authors declare that they have no relevant financial interests. 
Conclusions-Achieving the CMS goal of $66 \%$ prevalent AVF use is feasible for individual dialysis facilities. There is a need to reduce regional variation before the CMS goal can be fully realized for US hemodialysis facilities.

\section{INDEX WORDS}

Arterio-venous fistula; end-stage renal disease; hemodialysis

An arterio-venous fistula (AVF) is the preferred vascular access for hemodialysis (HD). The National Kidney Foundation's Kidney Disease Outcomes Quality Initiative (KDOQI) clinical practice guidelines for vascular access recommend pre-dialysis creation and use of an AVF at the onset of renal replacement therapy. ${ }^{1}$ The Fistula First Breakthrough Initiative (FFBI) was developed by the Centers for Medicare \& Medicaid Services (CMS) to collect, analyze and disseminate information to improve AVF use in the United States (US),2 similar to registry-based programs in Asia, Europe and South America.35 After attaining the initial FFBI goal of $40 \%$ prevalent AVF use, 6 CMS established a new national quality goal of 66\% AVF use among prevalent patients by June of 2009. The rationale for this new target was the observation that AVF use among HD patients in Europe and Asia varied between $60 \%$ and $90 \%{ }^{7}$

The international comparisons used to select the 2009 US prevalent AVF goal show substantial variability and it is not clear that these Asian and European end-stage renal disease (ESRD) populations are appropriate comparators for the US dialysis population. Further, AVF performance in the US is measured for the entire ESRD population, whereas international estimates are often based on treatment facility samples which may not fully capture care in the ESRD population. These considerations have led to questions about the appropriateness of the CMS goal for prevalent AVF use in the US HD population. This report examines the current level of achievement in reaching the FFBI goal using recent population-based data collected during the FFBI.

\section{METHODS}

\section{Study setting}

The 18 regional ESRD Networks comprise a surveillance system that collects, analyzes, and disseminates information about the patterns and outcomes of ESRD care in the US. The Networks are responsible for conducting the FFBI and, in collaboration with dialysis facilities, nephrologists and other health professionals, improving the use of AVFs by US HD patients. Networks are CMS contractors responsible for communicating the advantages of AVF use, providing improvement strategies and resources, performing education, and facilitating regional coalitions of stakeholders focused on quality improvement. All FFBI eligible facilities are FFBI participants.

\section{Study population}

The study population included all eligible HD facilities. Facilities which may be deemed ineligible by CMS on a case-by-case basis and, consequently, do not submit data are: (1) acute care hospital facilities, (2) Veterans' Administration facilities, (3) pediatric facilities, (4) permanently or temporarily closed facilities, (5) facilities providing only peritoneal dialysis services, and (6) special purpose facilities. All eligible facilities during the period January 2007 to April 2010 that met other criteria as described below were included. 


\section{Data and data collection}

Monthly data collected from each facility by a regional Network included the number of prevalent HD patients, and those with a vascular access that was an: AVF, central venous catheter (CVC) with an AVF present, CVC with an arterio-venous graft (AVG) present, $\mathrm{CVC} \geq 90$ days with no other access present, and $\mathrm{CVC}<90$ days with no other access present. All categories are mutually exclusive. The four CVC categories sum to the total number of patients using a CVC.

Monthly census data were collected by each dialysis facility using either an electronic workbook provided by the Network for return or a direct electronic submission for large dialysis organization. Large dialysis organizations are corporate entities that include 100 or more dialysis units. ${ }^{8}$ Included in the vascular access census data were counts of the type of vascular access used at each patient's last monthly hemodialysis treatment. We restricted our analysis to those facilities with an average patient census of 10 or more throughout the study period and data for all 40 months.

While patient demographic and clinical data are not collected as a function of the FFBI, data from the CMS Medical Evidence Form (CMS-2728), which is collected at the start of maintenance dialysis, were available for access at the end of the 40 month study period on June 1,2010. These data were used to construct a data set of all living prevalent in-center HD patients. Linking these patients with their current dialysis provider resulted in facilitylevel measures of patient demographic and clinical characteristics.

\section{Measures}

We measured AVF and CVC use at the facility level in the following manner: the denominator for all measures was the number of prevalent HD patients treated during the last treatment day of the calendar month. The following numerators apply:

1. AVF use (number of prevalent HD patients in the denominator who were dialyzed using an AVF on the last treatment day of the calendar month);

2. CVC $<90$ days use (number of prevalent HD patients in the denominator who were dialyzed using a CVC on the last treatment day of the calendar month; the CVC was the sole access in place and used for fewer than 90 days);

3. $\mathrm{CVC} \geq 90$ days use (number of prevalent $\mathrm{HD}$ patients in the denominator who were dialyzed using a CVC on the last treatment day of the calendar month; the CVC was the sole access in place and used for treatment for 90 days or greater);

4. CVC with AVG use (number of prevalent HD patients in the denominator who were dialyzed using a CVC on the last treatment day of the calendar month; patients had an AVG but were not using it)

5. CVC with AVF use (number of prevalent HD patients in the denominator who were dialyzed using a CVC on the last treatment day of the calendar month; patients had an AVF but were not using it);

6. Total CVC use (number of prevalent $\mathrm{HD}$ patients in the denominator who were dialyzed using a CVC on the last treatment day of the calendar month).

Target achievement was defined as the percent of facilities within the geographic boundaries of the entity being assessed (i.e., US or Network) with $66 \%$ or more prevalent HD patients using an AVF. 


\section{Statistical analysis}

Network descriptive statistics including facility casemix and other measures were prepared. To examine the longitudinal data set, we calculated the mean US and Network prevalent vascular access use for each of the 40 months. In addition, we calculated the percent of US and Network facilities achieving the CMS target at initial measurement in January 2007, at re-measurement in April 2010, and at least once over the 40 months. Mean changes in AVF and $\mathrm{CVC}$ use for the nation and Networks were tested using paired t-tests. Changes in the percent of facilities achieving the $66 \%$ prevalent AVF target were tested using McNemar's test for marginal homogeneity. Sustainability was examined through calculation of the mean number of months at target for those achieving the target at least once in 40 months.

To determine if there was a temporal effect, the study period was divided into four 10 month periods. For each study facility and time period, AVF use was regressed on time to produce average rates of change. Differences across the four periods were examined through analysis of variance.

To understand factors associated with AVF use at the facility and Network levels, two additional analyses were performed. First, facilities were divided into quintiles based on the proportion of patients using an AVF in the last measured month and differences in casemix and other facility characteristics were examined across performance quintiles using the nonparametric Kruskal-Wallis test. Second, the proportion of patients using an AVF in the last measured month was regressed on these same facility level measures. In order to examine the relationship between changes in AVF and total CVC use, the Spearman correlation coefficient was calculated. All analyses were performed using SAS software, version 9.1 (SAS Institute Inc, www.sas.com).

\section{RESULTS}

There were 5,692 facilities submitting data into the CMS vascular access database for January 2007 to April 2010, and 4,064 had an average patient census of 10 or more and data for all 40 months. Six of these facilities were excluded due to coding anomalies (i.e., having more than one Network number). Of the remaining 4,058 facilities included in our analyses, the mean (SD) patient census was 72.3 (42.4). Table 1 provides a description of facility casemix and other characteristics across Networks. AVF use improved in all Networks (Table 2 and Figure 1). In January 2007, the mean AVF use among all facilities was 45.3\% and increased to 55.5\% in April $2010(P<0.001)$, a 22.5\% increase over initial measurement. Each Network increased AVF use during that interval, with a Network mean (SD) percent increase in AVF use of $22.1 \%$ (5.6), and a median of $22.2 \%\left(25^{\text {th }}-75^{\text {th }}\right.$ percentile, $19.3 \%-24.7 \%)$.

Over the 40 months, 1,458 facilities (35.9\%) reached the CMS goal at least once. Attainment of the goal varied from $22.1 \%$ of all treatment facilities in Network 5 to $79.0 \%$ in Network 16 (Table 2 and Figure 2). The mean (SD) percent of facilities within a Network attaining the AVF goal at least once during that interval was $38.7 \%$ (15.0), with a median of $36.7 \%\left(25^{\text {th }}-75^{\text {th }}\right.$ percentile, $\left.26.1 \%-47.9 \%\right)$. Facilities achieving the prevalent AVF use target at least once maintained the target for a mean (SD) of 12.9 (11.7) months.

Improvement continued throughout the 40 month interval. Table 3 provides mean facility rates of change over four ten month periods. Some slowing of improvement was evident in later periods, coinciding with higher mean AVF use. Through pairwise comparisons using Tukey's procedure and $\alpha=.05$, the average rate of change in the initial time period was found to be significantly different from those in other periods. 
The proportion of facilities with $66 \%$ or more of their patients using an AVF increased from $6.4 \%$ during January 2007 to $19.0 \%$ during April $2010(P<0.001)$. The proportion of treatment facilities attaining 66\% AVF use in April 2010 varied by Network, from a low of $9.4 \%$ of Network 6 facilities to a high of $49.2 \%$ of facilities in Network 16 (Table 2 and Figure 3). The Network mean (SD) increase in facilities reaching goal in a particular month was $414.7 \%$ (652.5), with median of $218.3 \%\left(25^{\text {th }}-75^{\text {th }}\right.$ percentile, $\left.145.5 \%-337.5 \%\right)$. The geographic variations implicit in the Network differences in AVF use showed little pattern. Although there was a contiguous group of Networks (5, 6, 8, 9, and 10) with lower percentages of facilities accomplishing the $66 \%$ prevalent AVF use goal in April 2010, there were also contiguous Networks with high and low proportions of facilities achieving the goal in that time period. (Figure 2.)

Examination of facility casemix measures across rising AVF use quintiles, per the last measured month, revealed declining median percentages of females, African Americans, and those with hypertension as a cause of ESRD. (Table 4.) All other median casemix measures increased over increasing quintiles. While there were significant differences across quintiles in facility size, as measured by the prevalent HD patient population, and the percentage of LDO facilities, no trend was apparent. Facility vascular access measures varied in an expectable manner given the way the quintiles were constructed.

Linear regression, with an R2 of 0.20, revealed lower percentages of females, younger average age, and smaller percentages of patients with peripheral vascular disease at the start of chronic HD associated with higher AVF use. Higher percentages of patients who were Caucasian, Hispanic, and had diabetes or glomerulonephritis as a primary cause of ESRD were associated with higher AVF use. Higher percentages of patients beginning HD with an AVF and being an large dialysis organization facility were also associated with higher prevalent AVF use (Table 5).

As AVF use increased within each Network, CVC use declined (Table 2 and Figure 4). Nationally, total CVC use declined over the 40 month period from $28.8 \%$ in January 2007 to $24.0 \%$ in April $2010(P<0.001)$, a 16.4\% decrease. Total CVC use also declined in each Network with a mean (SD) percent change in total CVC use of $-16.2 \%(2.5)$, with a median of $-16.3 \%\left(25^{\text {th }}-75^{\text {th }}\right.$ percentile, $-17.8 \%$ to $\left.-14.7 \%\right)$.

CVC use declined in each category (Table 6). The mean chronic CVC use (CVC $\geq 90$ days) declined the most, from $12.0 \%$ to $9.5 \%$. Finally, the change in AVF use was inversely correlated with the change in total CVC use $(\rho=-0.5791)$.

\section{DISCUSSION}

While the CMS goal of $66 \%$ prevalent AVF use has not yet been reached nationally, our observations, which are based on national, monthly reporting by all FFBI eligible facilities in the US, show that the goal is frequently attained by individual facilities; however, success varies substantially among facilities in different Networks. Because of the shared responsibility for improvement by facilities and Networks, it is useful to examine progress and consider additional strategies for improvement.

There is ample evidence to support the US efforts to increase AVF use. An AVF requires fewer interventions for access dysfunction, has better four to five year patency rates, and is associated with lower morbidity and mortality. $1,9,10,11$ In addition, costs are lower, with an average 2007 per person per year total cost for a patient with a CVC of $\$ 79,364$; for a patient with an AVG, $\$ 72,729$; and for a patient with an AVF, just under $\$ 60,000{ }^{8}$ 
What has not been clear is the extent to which successful AVF use can be achieved within different HD populations. Our analysis demonstrates that AVF use of $66 \%$ can be attained and sustained by many US facilities. In our study, 1,458 facilities were able to achieve the AVF use target at least once during the observation period and these facilities sustained the target, on average, for almost 13 months. Improvement in AVF use and goal attainment were observed among facilities in all Networks regardless of initial measurement. These observations suggest that additional increases in AVF use are attainable and that an AVF use of $66 \%$ will be attained by some Networks and many facilities serving the US HD population.

An important aspect of this analysis is the facility-to-facility variation in AVF goal achievement within individual ESRD Networks. The proportion of Network facilities in April 2010 that achieved the AVF goal varied between $9.4 \%$ and $49.2 \%$ of all facilities. At a population level this is associated with respective AVF use of $51.6 \%$ and $64.8 \%$ of all HD patients. Clearly, it is important to understand why vastly different performance is observed, often in geographically contiguous Networks.

Patient casemix explained $20 \%$ of the variance in our analysis. Facilities with higher percentages of Caucasian and Hispanic patients, patients with diabetes as the primary cause of ESRD, patients with glomerulonephritis as the primary cause, and patients beginning chronic HD with an AVF tended to have higher prevalent AVF use. Perhaps an explanation for the positive associations between AVF use and percentages of patients with diabetes and glomerulonephritis is that these patients are more likely to receive pre-ESRD care and preparation for a permanent vascular access, even if they do not begin with an AVF. It is obvious that receiving higher percentages of patients with an AVF at initiation predisposes a facility to achieving higher prevalent AVF use. The higher AVF use in LDO facilities may be facilitated by corporate programs promoting chronic $\mathrm{CVC}$ reduction.

Our analysis also revealed lower AVF use among facilities with higher percentages of females and patients starting chronic HD with PVD and older populations. These are patients for whom constructing and maintaining an AVF may be more challenging. However, it is cautionary to remember that the slogan "Fistula First" was never intended to suggest that all patients should receive an AVF. ${ }^{6}$ Patients with a high likelihood of AVF non-maturation or short life expectancy, for example, may not be appropriate candidates for an AVF and may benefit from an AVG, which has the benefit of reducing the proportion of CVC dependent patients. However, maturation risk algorithms ${ }^{12}$ should not be used to eliminate patients from consideration for an AVF. All patients should be evaluated by a skilled surgeon and considered for placement of an AVF unless medically contraindicated.

In our analysis, casemix explained only a portion of the variation in facility-level AVF use. We do not yet fully understand regional variability. Practice patterns reflecting preferences or skill level of the surgeons, nephrologists, and dialysis unit staff have been implicated in geographic variation.13 Other potential reasons for geographic differences include variability in the timeliness of patient referrals to nephrologists; the number of surgeons willing and able to perform AVF surgery; the length of time to surgical referral; AVF primary failure rates; successful cannulation of AVFs by dialysis staff; the number of patients without usable veins due to prolonged CVC use and central vein occlusion; and insurance coverage and funding structure. For example, in the Dialysis Access Consortium (DAC) study, $61 \%$ of AVFs failed to mature. ${ }^{14}$ Additionally, funding structure may differ by state (i.e. patients with no insurance may have access to vascular surgeons at a county hospital, while others may not) and state Medicaid coverage varies. 
Understanding the nature of differences and what can be done to reduce variation while simultaneously improving performance is imperative. In this regard, it is important to emphasize that the regional variability represented by differences across US ESRD Networks are similar to national variations in AVF use reported by the Dialysis Outcomes and Practice Patterns Study (DOPPS). ${ }^{7}$ Factors that contribute to these within and between country variations in quality of care are also poorly understood and warrant attention.

This analysis also demonstrates that the rise in AVF use since 2007 has not been accompanied by a corresponding increase in CVC use, which trended down significantly over the 40 months of study observation. These findings corroborate those of Spergel, 6 who examined data from $2003-2008$, and refuted pronouncements in the literature that the FFBI initiative has led to an increase in CVC use. ${ }^{15}$ The significant decline in CVC use was observed despite the fact that the overwhelming majority of initial start HD patients continue to begin chronic dialysis with a CVC $\left(81.8 \%\right.$ in 2009). ${ }^{16}$ Thus, it appears that facilities are successfully converting CVCs to permanent vascular accesses and assuring that patients do not dialyze greater than 90 days with a CVC without placing a permanent access. In fact, the prevalent CVC $\geq 90$ days use reported here was $10.5 \%$ in April 2010, comparing favorably to the K/DOQI guidelines for $10 \%$ or fewer HD patients to dialyze with a CVC greater than 90 days.

To further improve AVF use while decreasing variation, several actions may be pursued. These include utilization of a vascular access nurse in the dialysis clinic; defining and utilizing criteria for early AVF assessment and intervention if not maturing; evaluating the impact of cannulation policy for dialysis staff to aid in AVF preservation; and educating patients and nephrologists to the dangers of CVCs. For example, by implementing a program which included a dedicated vascular access nurse to coordinate patient care and an algorithm to prioritize AVF surgery, one dialysis facility documented an increase in AVF use from $56 \%$ to $75 \% .{ }^{17}$ In the DAC study, $2 \%$ of non-maturing AVFs were referred for intervention to assist maturation before six weeks. ${ }^{18}$ Factors such as adequate length of the AVF cannulation segment and use of the buttonhole cannulation technique may reduce cannulation-related complications. ${ }^{19}$ In a review by Rehman, et al, the authors concluded that "nephrologists are ethically obligated to systematically explain to patients the harms of tunneled cuffed catheters." 20 As rationale, they cited the increased risk of death, increased risk of serious infection, increased hospitalization, a decreased likelihood of adequate dialysis, and an increased number of vascular access procedures associated with long-term catheter use.

But, who should take responsibility for assuring that all of the above actions occur? An expert panel recently convened by CMS concluded that the highest priority strategy for improving AVF use and reaching the CMS goal is "Nephrology Leadership." ${ }^{1}$ According to these experts, not only does the nephrologist have an ethical obligation to promote catheter reduction, but he/she should provide the necessary leadership to assure that patients are prepared for dialysis, that prescriptions are appropriate and that quality outcomes are achieved. The nephrologist has the primary responsibility for assuring that patients understand the superior benefits of AVFs, patients are referred to surgeons who are technically competent to place AVFs, staff is trained to cannulate AVFs, and surveillance and monitoring protocols are in place to track maturing AVFs and refer when appropriate.

While this analysis is limited by the absence of facilities' case-mix data over time and the national scope of the initiative precludes a comparison group, it demonstrates that the CMS goal of $66 \% \mathrm{AVF}$ use among prevalent HD patients is achievable, without a concurrent rise in CVC use, as demonstrated by a significant number of dialysis facilities across the US; 
however, challenges remain. There is a need to further spread best clinical practices to reduce regional variation and fully realize the CMS goal. This is the role of Networks.

\section{Acknowledgments}

The content of this publication does not necessarily reflect the views or policies of the Department of Health and Human Services, nor does mention of trade names, commercial products, or organizations imply endorsement by the U.S. Government.

Support: The analyses upon which this publication is based were performed under Contract Number 500-06NW005C entitled End Stage Renal Disease Networks Organization for the District of Columbia, Maryland, Virginia and West Virginia, sponsored by the Centers for Medicare \& Medicaid Services, Department of Health and Human Services. This article is a direct result of the Health Care Quality Improvement Program initiated by the Centers for Medicare \& Medicaid Services, which has encouraged identification of quality improvement projects derived from analysis of patterns of care, and therefore required no special funding on the part of this contractor. This work was also supported in part by a National Institutes of Health Career Development Award K23 DK65634 (to Dr Wasse).

\section{REFERENCES}

1. National Kidney Foundation. KDOQI clinical practice guidelines and clinical practice recommendations for vascular access 2006. Am J Kid Dis 2006;48(suppl 1):s176-s322. [PubMed: 16813989]

2. McClellan WM, Krisher JO. Collecting and using patient and treatment center data to improve care: adequacy of hemodialysis and end-stage renal disease surveillance. Kidney Int 2000;57(suppl 74):s7-s13.

3. Teck-Onn L, Yam-Ngol L, Morad Z. Review article: Use of renal registry data for research, healthcare planning and quality improvement: what can we learn from registry data in the Asia-Pacific region? Nephrology 2008;13(8):745-752. [PubMed: 19154324]

4. Will EJ. Renal registries in the era of guidelines, standards and quality improvement. One view from the UK Renal Registry experience. J Nephrol 2006;19(4):492-499. [PubMed: 17048207]

5. Steil H, Amato C, Carioni C, et al. EuCliD—a medical registry. Methods Inf Med 2004;43(1):8388. [PubMed: 15026844]

6. Spergel LM. Has the Fistula First Breakthrough Initiative caused an increase in catheter prevalence? Semin Dial 2008;21(6):550-5522. [PubMed: 19000118]

7. Ethier J, Mendelssohn DC, Elder SJ, et al. Vascular access use and outcomes: an international perspective from the Dialysis Outcomes and Practice Patterns Study. Nephrol Dial Transplant 2008;23(10):3219-3226. [PubMed: 18511606]

8. US Renal Data System. USRDS 2009 Annual Data Report: Atlas of Chronic Kidney Disease and End-Stage Renal Disease in the United States, National Institutes of Health, National Institute of Diabetes and Digestive and Kidney Diseases. Bethesda, MD: 2009.

9. Allon M. Current management of vascular access. Clin J Am Soc Nephrol 2007;2:786-800. [PubMed: 17699495]

10. Astor BC, Eustance JA, Powe NR, et al. Type of vascular access and survival among incident hemodialysis patients: the choices for healthy outcomes in caring for ESRD (CHOICE) study. J Am Soc Nephrol 2005;16(5):1449-1455. [PubMed: 15788468]

11. Xue JL, Dahl D, Ebben JP, Collins AJ. The association of initial access type with mortality outcomes in elderly Medicare ESRD patients. Am J Kidney Dis 2003;42(5):1013-1019. [PubMed: 14582045]

12. Lok CE, Allon M, Moist L, Oliver MJ, Shah H, Zimmerman D. Risk equation determining unsuccessful cannulation events and failure to maturation in arteriovenous fistulas (REDUCE FTM I). J Am Soc Nephrol 2006;17(11):3204-3212. [PubMed: 16988062]

13. Allon M, Robbin M. Increasing arteriovenous fistulas in hemodialysis patients: problems and solutions. Kidney Int 2002;62:1109-1124. [PubMed: 12234281] 
14. Dember LM, Beck GJ, Allon M, et al. for the Dialysis Access Consortium Study Group. Effect of Clopidogrel on early failure of arteriovenous fistulas for hemodialysis: a randomized controlled trial. JAMA 2008;299(18):2164-2171. [PubMed: 18477783]

15. Lok CE. Fistula First Initiative: advantages and pitfalls. Clin J Am Soc Nephrol 2007;2:10431053. [PubMed: 17702726]

16. Fistula First Data page. Fistula First Web site. [July 21, 2010]. Available at http://www.fistulafirst.org/AboutFistulaFirst/FFBIData.aspx.

17. Polkinghorne KR, Seneviratne M, Kerr PG. Effect of a vascular access nurse coordinator to reduce central venous catheter use in incident hemodialysis patients: a quality improvement report. Am J Kidney Dis 2008;53(1):99-106. [PubMed: 18805614]

18. Van Loon MM, Kessels AGH, Van der Sande FM, Tordoir JHM. Cannulation and vascular accessrelated complications in hemodialysis: factors determining successful cannulation. Hemodialysis Int 2009;13(4):498-504.

19. Van Loon MM, Goovaerts T, Kessels AGH, van der Sande FM, Tordoir JHM. Buttonhole needling of hemodialysis arteriovenous fistulae results in less complications and interventions compared to the rope-ladder technique. Neph Dial Transp 2010;25(1):225-230.

20. Rehman R, Schmidt RJ, Moss AH. Ethical and legal obligation to avoid long-term tunneled catheter access. Clin J Am Soc Nephrol 2009;4(2):456-460. [PubMed: 19158368]

21. Fistula First Home page. Fistula First Web site. [July 21, 2010]. Available at http://www.fistulafirst.org/. 


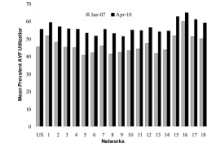

Figure 1.

Mean prevalent arterio-venous fistula use in hemodialysis facilities in the United States and the end-stage renal disease Networks, January 2007 and April 2010.

Source: Centers for Medicare \& Medicaid Services, Vascular Access Data Set as delivered by the Network Information Technology Support contractor to Fistula First Breakthrough Initiative contractor, January 2007 - April 2010. 


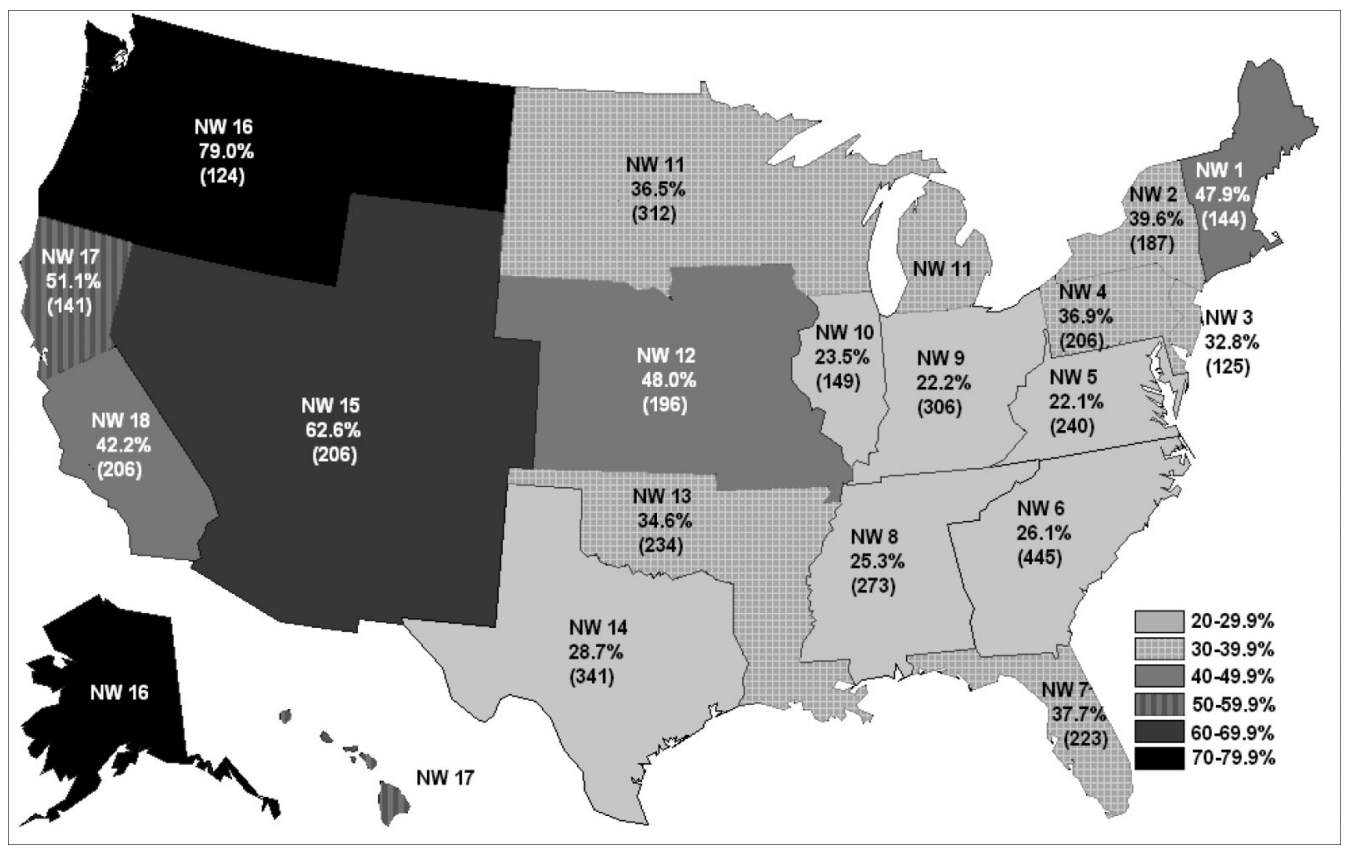

Figure 2.

Percent of hemodialysis facilities (with $\geq 10$ patients and 40 months of data) achieving the Centers for Medicare \& Medicaid Services Goal of $66 \%$ for prevalent arterio-venous fistula (AVF) use at least once between January 2007 and April 2010.

Source: Centers for Medicare \& Medicaid Services, Vascular Access Data Set as delivered by the Network Information Technology Support contractor to Fistula First Breakthrough Initiative contractor, January 2007 - April 2010. 
60 $\square$ Jan-07 $\quad$ Apr-10

50

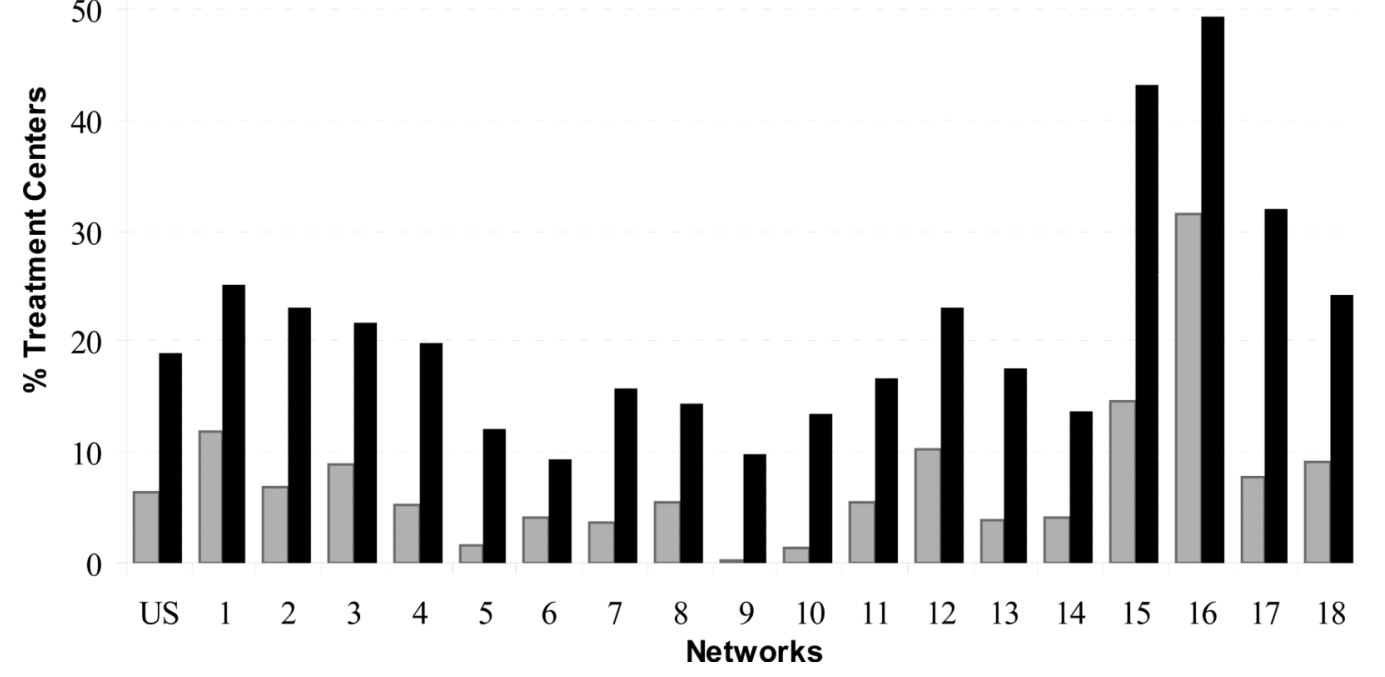

Figure 3.

Percent hemodialysis facilities in the United States and end-stage renal disease Networks achieving the Centers for Medicare \& Medicaid Services prevalent arterio-venous fistula (AVF) goal of 66\%, January 2007 and April 2010.

Source: Centers for Medicare \& Medicaid Services, Vascular Access Data Set as delivered by the Network Information Technology Support contractor to Fistula First Breakthrough Initiative contractor, January 2007 - April 2010. 


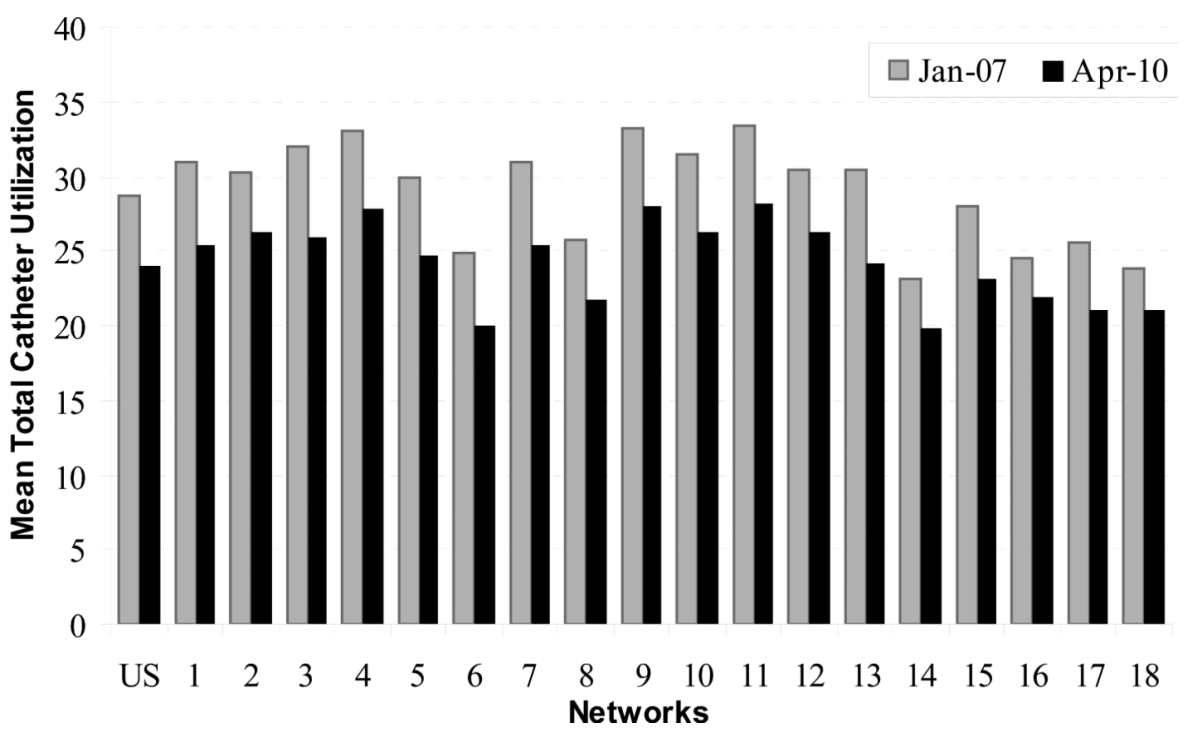

Figure 4.

Mean prevalent central venous catheter (CVC) use in hemodialysis facilities in the United States and the end-stage renal disease Networks, January 2007 and April 2010.

Source: Centers for Medicare \& Medicaid Services, Vascular Access Data Set as delivered by the Network Information Technology Support contractor to Fistula First Breakthrough Initiative contractor, January 2007 . April 2010. 


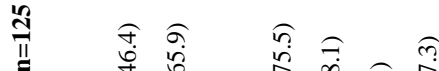

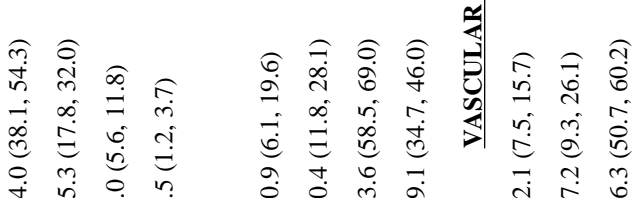

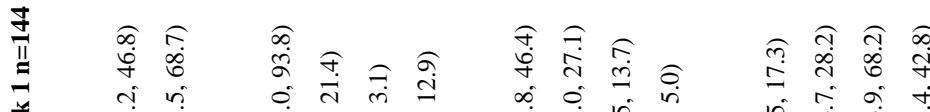

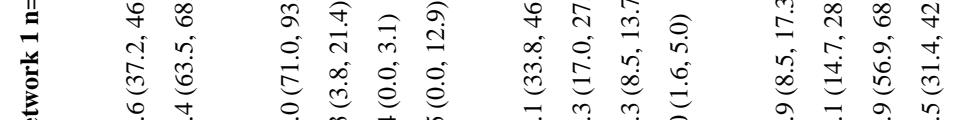

\section{m $\mathrm{y}=\dot{m}$}

ஸे ‡ 


\begin{tabular}{|c|c|c|c|c|c|c|c|c|c|c|c|c|c|c|c|c|c|c|c|}
\hline$\frac{\Delta}{N} \equiv \& \frac{0}{\Sigma}$ & के & $\begin{array}{l}\stackrel{2}{\sigma} \\
\dot{\sigma}\end{array}$ & $\begin{array}{l}0 \\
\stackrel{2}{\circ}\end{array}$ & $\begin{array}{l}\infty \\
\text { i } \\
m\end{array}$ & $\begin{array}{l}\hat{a} \\
\dot{\rho}\end{array}$ & $\overrightarrow{\vec{i}}$ & $\overrightarrow{\mathrm{i}}$ & $\stackrel{r}{\dot{m}}$ & \begin{tabular}{|c|}
$m$ \\
2 \\
2 \\
\end{tabular} & $\begin{array}{l}\tilde{N} \\
\text { ה }\end{array}$ & $\ddot{n}$ & $\begin{array}{l}n \\
n \\
n \\
n\end{array}$ & $\begin{array}{c}\stackrel{0}{\infty} \\
\stackrel{\infty}{+}\end{array}$ & $\begin{array}{l}\stackrel{\circ}{m} \\
\dot{m}\end{array}$ & $\underbrace{\vec{d}}_{\dot{d}}$ & $\begin{array}{l}0 \\
\text { i }\end{array}$ & ㅇ. & $\overline{\vec{n}}$ & $\tilde{z}$ \\
\hline $\begin{array}{l}\bar{T} \\
\text { Uू } \\
0^{\circ}\end{array}$ & $\vec{\infty}$ & $\begin{array}{l}\infty \\
\dot{\Xi} \\
\end{array}$ & $\begin{array}{l}\infty \\
\dot{0} \\
\stackrel{\tilde{\nu}}{0}\end{array}$ & $\mid \begin{array}{l}n \\
n \\
y \\
z\end{array}$ & $\mid \begin{array}{r}\vec{d} \\
\stackrel{a}{a}\end{array}$ & $\mid \begin{array}{l}0 \\
\text { तु } \\
\text { in }\end{array}$ & m. & 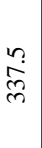 & $\dot{0}$ & $\left|\begin{array}{l}0 \\
\dot{0} \\
0 \\
i\end{array}\right|$ & 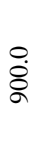 & aे. & $\begin{array}{l}0 \\
\grave{I} \\
\end{array}$ & $\begin{array}{l}0 \\
\dot{b} \\
\\
m\end{array}$ & तે & ळ. & $\begin{array}{l}+ \\
b \\
n\end{array}$ & & \\
\hline$\stackrel{g}{\Xi}$ & & $\mid \begin{array}{l}\vec{\delta} \\
\dot{0} \\
v\end{array}$ & $\begin{array}{l}\bar{\delta} \\
\dot{0} \\
v\end{array}$ & $\tilde{D}_{0}$ & $\mid \begin{array}{l}\vec{\delta} \\
\dot{0} \\
v\end{array}$ & $\mid \begin{array}{l}\vec{\delta} \\
\dot{0} \\
v\end{array}$ & $\mid \begin{array}{l}\bar{\delta} \\
\dot{\theta} \\
v\end{array}$ & $\begin{array}{l}\overline{0} \\
0\end{array}$ & $\mid \begin{array}{c}\vec{\delta} \\
\dot{0} \\
\dot{v}\end{array}$ & $\mid \begin{array}{l}\bar{o} \\
\dot{0} \\
\dot{v}\end{array}$ & $\begin{array}{l}\bar{\delta} \\
\dot{0} \\
\text { v }\end{array}$ & $\begin{array}{l}\bar{\delta} \\
\dot{0} \\
v\end{array}$ & $\vec{\sigma}$ & $\begin{array}{l}\vec{b} \\
\dot{0} \\
v\end{array}$ & $\begin{array}{l}\bar{b} \\
\dot{0} \\
\dot{0}\end{array}$ & $\begin{array}{l}\bar{\delta} \\
\dot{\theta} \\
v\end{array}$ & & & \\
\hline
\end{tabular}

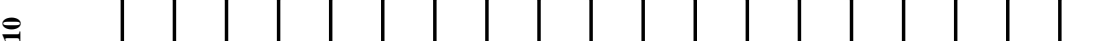

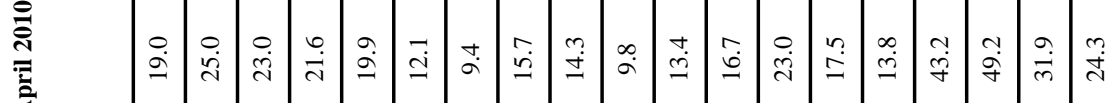

䒽

\section{(1)}

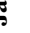

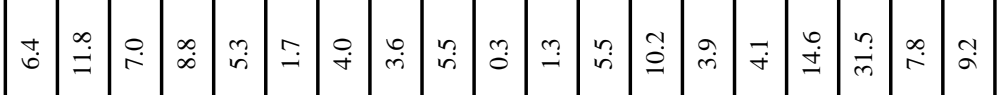

总

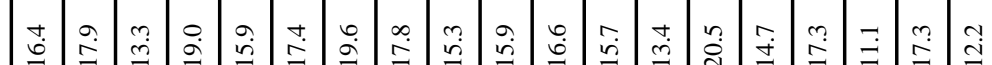

$\div \div$

爱

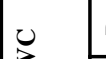

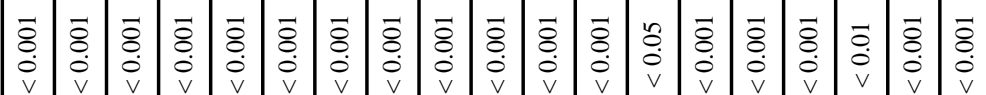



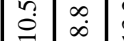

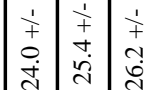

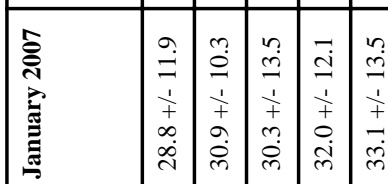

$\stackrel{\mathscr{\omega}}{:}$

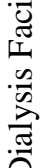

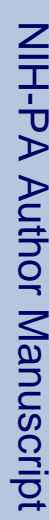

西

离

范

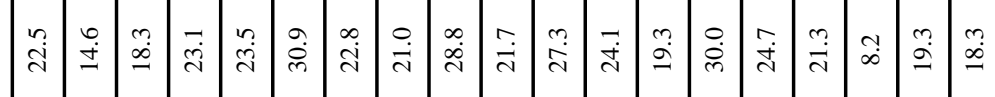

స్

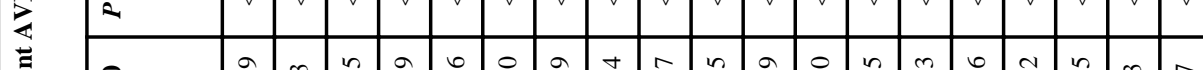

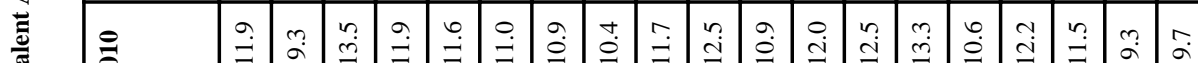

焉

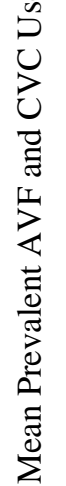

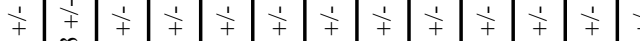

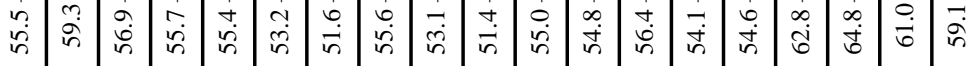

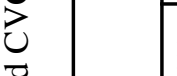


Table 3

Mean Facility Rates of Change in AVF Use by Time Period

\begin{tabular}{|lcccc|}
\hline & $\begin{array}{c}\text { Time Period 1(Jan 07 - Oct } \\
\mathbf{0 7} ; \mathbf{n}=\mathbf{4 , 0 5 8})\end{array}$ & $\begin{array}{c}\text { Time Period 2 (Oct 07 - Aug } \\
\mathbf{0 8} ; \mathbf{n}=\mathbf{4 , 0 5 8})\end{array}$ & $\begin{array}{c}\text { Time Period 3 (Aug 08 - Jun } \\
\mathbf{0 9} ; \mathbf{n}=\mathbf{4 , 0 5 8})\end{array}$ & $\begin{array}{c}\text { Time Period 4 (Jun 09 - Apr } \\
\mathbf{1 0} ; \mathbf{n}=\mathbf{4 , 0 5 8})\end{array}$ \\
\hline Mean & 0.34 & 0.28 & 0.22 & 0.24 \\
SD & 0.9 & 0.8 & 0.8 & 0.8 \\
\hline
\end{tabular}

$\mathrm{F}=17.6 ; d f=3 ; P<0.001$

Source: Centers for Medicare \& Medicaid Services, Vascular Access Data Set as delivered by the Network Information Technology Support contractor to Fistula First Breakthrough Initiative contractor, January 2007 - April 2010.

AVF, arteriovenous fistula 


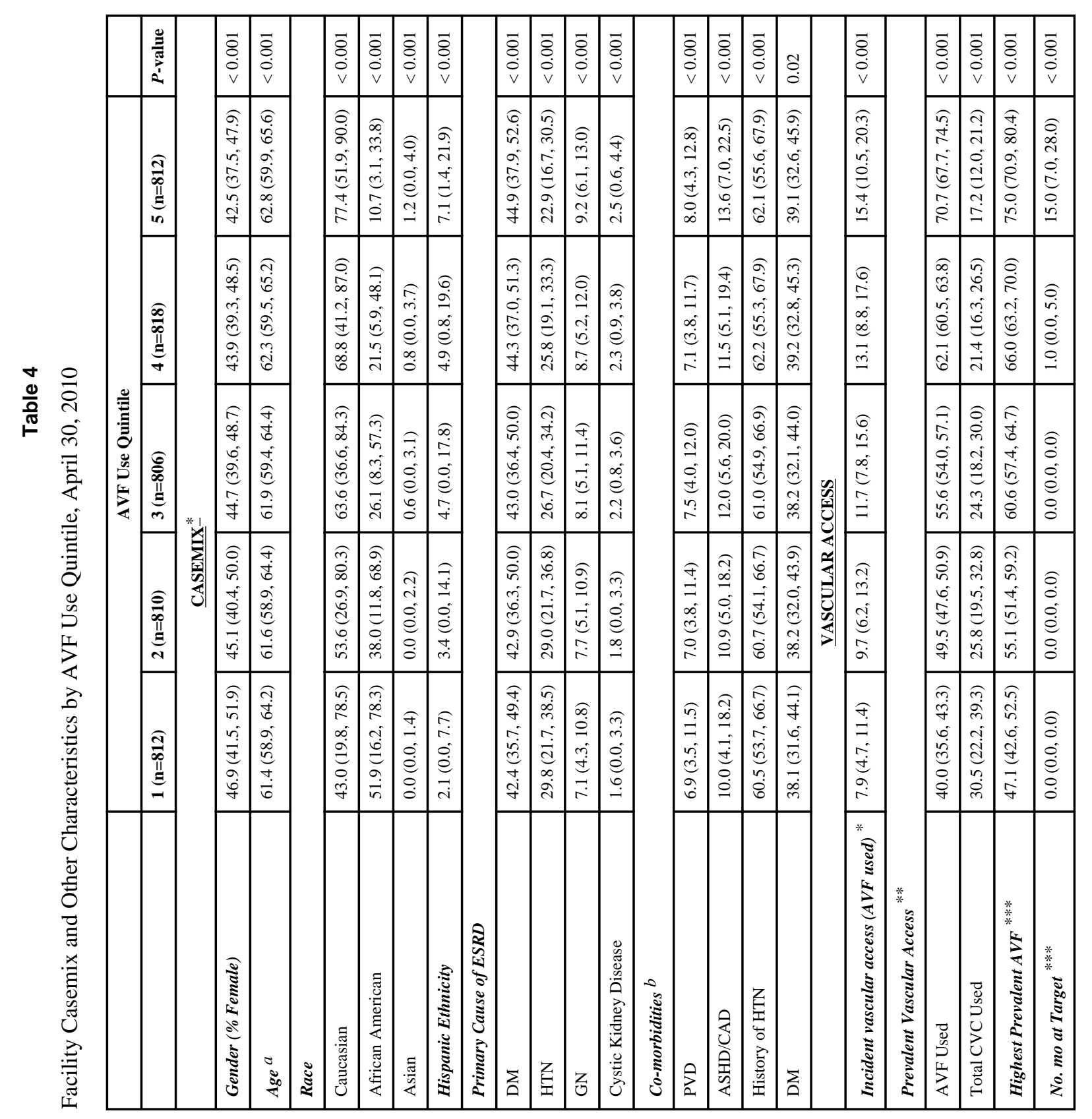




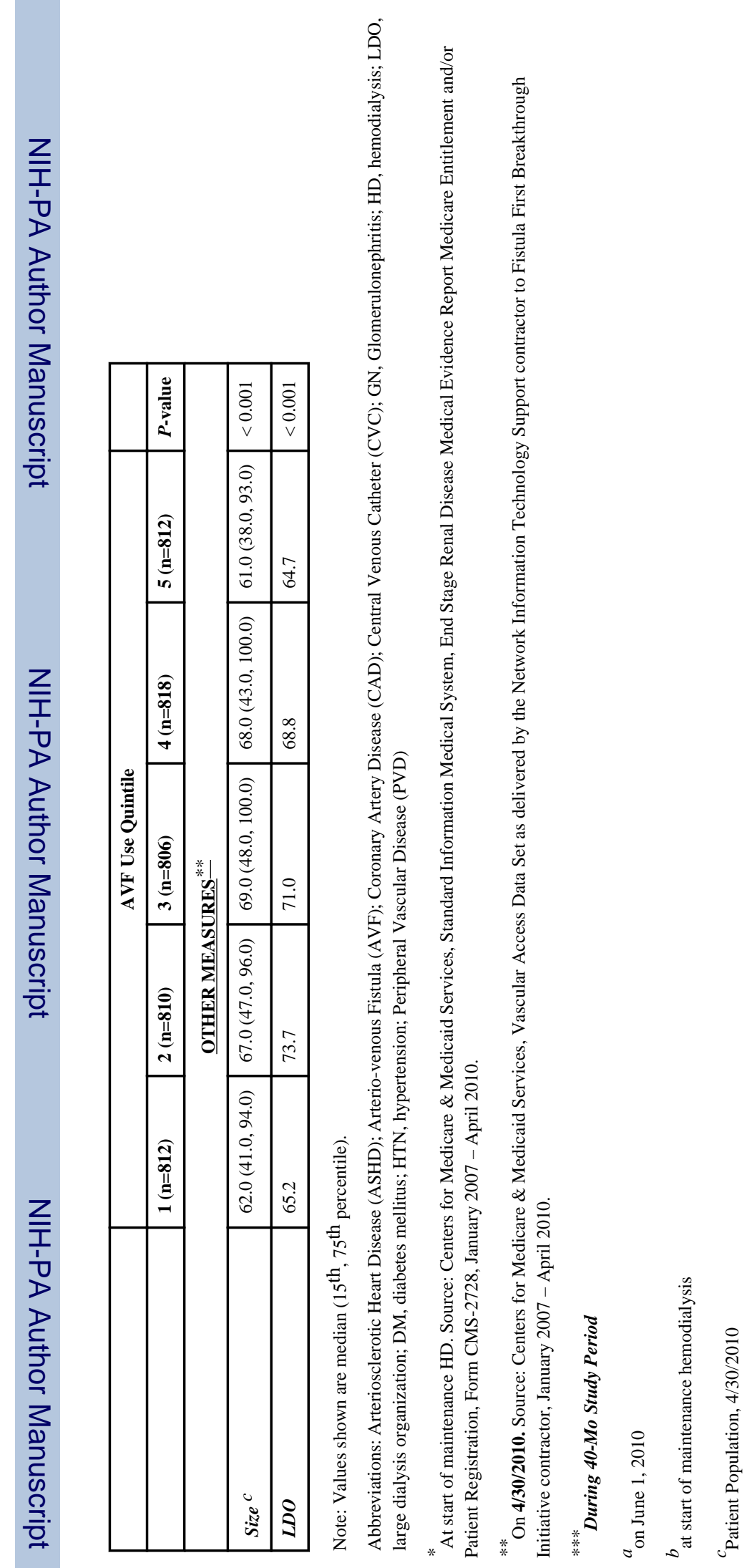


Table 5

Facility AVF Use as a Function of Casemix and Other Facility Characteristics

\begin{tabular}{|l|l|l|}
\hline Variable & $\boldsymbol{\beta}$ & $\boldsymbol{P}$-value \\
\hline Intercept & 55.868 & $<0.001$ \\
\hline Percent Female & -0.135 & $<0.001$ \\
\hline Average Age & -0.149 & 0.001 \\
\hline Percent Caucasian & 0.058 & $<0.001$ \\
\hline Percent Hispanic & 0.039 & $<0.001$ \\
\hline Percent Diabetes as Primary Cause & 0.087 & 0.001 \\
\hline Percent Hypertension as Primary Cause & 0.005 & 0.8 \\
\hline Percent Glomerulonephritis as Primary Cause & 0.144 & $<0.001$ \\
\hline Polycystic Kidney Disease as Primary Cause & -0.078 & 0.2 \\
\hline Percent PVD Co-morbidity & -0.086 & 0.005 \\
\hline Percent ASHD or IHD Co-morbidity & -0.005 & 0.8 \\
\hline Percent Hypertension Co-morbidity & -0.023 & 0.3 \\
\hline Percent Diabetes Co-morbidity & -0.019 & 0.4 \\
\hline Percent using AVF at Start of Maintenance HD & 0.652 & $<0.001$ \\
\hline Facility HD patients & 0.007 & 0.1 \\
\hline Large Dialysis Organization & 0.760 & 0.03 \\
\hline F value & 69.25 & $<0.001$ \\
\hline R $^{2}$ & 0.202 & \\
\hline
\end{tabular}

Source: Centers for Medicare \& Medicaid Services, Standard Information Medical System, End Stage Renal Disease Medical Evidence Report Medicare Entitlement and/or Patient Registration, Form CMS-2728, January 2007 - April 2010. Source for facility HD patients and LDO data:

Centers for Medicare \& Medicaid Services, Vascular Access Data Set as delivered by the Network Information Technology Support contractor to Fistula First Breakthrough Initiative contractor, January 2007 - April 2010.

AVF, arteriovenous fistula; Peripheral Vascular Disease (PVD); Atherosclerotic Heart Disease (ASHD); Ischemic Heart Disease (IHD); Hemodialysis (HD) 
Table 6

Mean Changes in US Prevalent CVC and AVF Use, January 2007 - April 2010

\begin{tabular}{|l|c|c|c|c|}
\hline Vascular Access & January 2007 (\%) & April 2010 (\%) & Difference (95\% CI) & $\boldsymbol{P}$-value \\
\hline CVC & & & & \\
\hline$\geq 90$ days & 12.0 & 9.5 & $-2.5(-2.7,-2.2)$ & $<0.001$ \\
\hline$<90$ days & 6.8 & 5.4 & $-1.4(-1.6,-1.2)$ & $<0.001$ \\
\hline with AVF & 7.9 & 7.5 & $-0.4(-0.6,-0.2)$ & $<0.001$ \\
\hline with AVG & 2.1 & 1.7 & $-0.5(-0.6,-0.4)$ & $<0.001$ \\
\hline Total & 28.8 & 24.0 & $-4.7(-5.0,-4.4)$ & $<0.001$ \\
\hline AVF & 45.3 & 55.5 & $10.2(9.8,10.5)$ & $<0.001$ \\
\hline
\end{tabular}

Source: Centers for Medicare \& Medicaid Services, Vascular Access Data Set as delivered by the Network Information Technology Support contractor to Fistula First Breakthrough Initiative contractor, January 2007 - April 2010.

Arterio-venous Fistula (AVF); AVG, arteriovenous graft; Central Venous Catheter (CVC) 